\title{
COMPARISON OF FOREST STRUCTURE METRICS DERIVED FROM UAV LIDAR AND ALS DATA
}

\author{
M. Bruggisser ${ }^{1 *}$, M. Hollaus ${ }^{1}$, D. Kükenbrink ${ }^{2}$, N. Pfeifer ${ }^{1}$ \\ ${ }^{1}$ Department of Geodesy and Geoinformation, TU Wien, 1040 Vienna, Austria - \\ (moritz.bruggisser, markus.hollaus, norbert.pfeifer)@geo.tuwien.ac.at \\ ${ }^{2}$ Remote Sensing Laboratories, Department of Geography, University of Zurich, 8057 Zurich, Switzerland - \\ daniel.kuekenbrink@geo.uzh.ch
}

\begin{abstract}
KEY WORDS: Point height distribution, Fractional cover, Vertical complexity index, Voxel metrics, Occlusion effect, Acquisition
\end{abstract} characteristics

\begin{abstract}
:
Point clouds derived from airborne laser scanning (ALS) and from LiDAR sensors mounted on unmanned aerial vehicles (ULS) reveal differences caused by the different sensor systems and acquisition geometries. These differences in the system characteristics are reflected in forest structure metrics that are derived from the respective point clouds. In our study, we investigate the completeness of scene coverage between the two systems and address differences between structure metrics derived from ULS and ALS, namely in point height quantiles, fractional cover $(f c)$, the vertical complexity index $(V C I)$ and the number of canopy layers $(n L a y e r s)$. The metrics are evaluated for raster cell sizes of $1-10 \mathrm{~m}$ in order to investigate the spatial scale on which the sensor systems provide comparable metrics. We found highest correspondences between ALS and ULS in the VCI- and the $n$ Layers-metrics, while $f c$ revealed large differences. For the height quantiles, the absolute differences were larger for the 10\%- (h10) and the 50\%- (h50) than for the 90\%- (h90) height quantile. Furthermore, we found differences between ALS- and ULS-metrics to decrease for larger cell sizes, except for $f c$, for which the differences increased, and $h 50$ and $h 90$, respectively, for which the differences were relatively stable for all cell sizes.
\end{abstract}

\section{INTRODUCTION}

Unnmanned aerial vehicles (UAV) mounted laser scanning systems, hereafter referred to as ULS, facilitate the derivation of forest structure information for forest inventories (FI) (Guo et al., 2017; Wallace et al., 2014, 2016). FI-parameters traditionally have been retrieved from ALS on an operational level (Næsset, 2014; Vauhkonen et al., 2014; White et al., 2016). In comparison to ALS, ULS acquisitions provide much higher point densities that are comparable to the ones from terrestrial laser scanning (Morsdorf et al., 2017; Wieser et al., 2017), what allows for the retrieval of FI-parameters on single tree level (Lin et al., 2011; Wallace et al., 2014; Wieser et al., 2017).

ULS and ALS acquisition campaigns differ broadly regarding the areas, which are typically covered, the overall costs of the missions, and the costs per square kilometer. While the costs per square kilometer are higher for ULS than for ALS data (factor 30-40 in the data sets in this study), the overall costs of typical ULS campaigns are lower compared to ALS acquisitions as their spatial extent typically is limited to local scales (e.g. Morsdorf et al., 2017; Wallace et al., 2014; Wieser et al., 2017). Yet, a comparison of the costs is also difficult because ULS can partly substitute in-situ measurements and then the relation of the costs is completely different. Furthermore, one can expect that prices for ULS acquisitions will decrease with the developing markets. For these reasons, ULS is a promising tool for the collection of LiDAR time series (Goodbody et al., 2017; Wallace et al., 2014; Wieser et al., 2017), providing the potential of vegetation monitoring and the update of FIs with a fine temporal resolution. ULS data in this context could complement data sets from ALS acquisitions, which are typically performed through the local authorities at temporal intervals of several years (Hollaus, 2015).

\footnotetext{
${ }^{*}$ Corresponding author
}

However, if metrics derived from point clouds from different laser scanning sources are to be combined, their comparability is a prerequisite. For ALS data, the effect of different acquisition characteristics on derived FI-metrics has been investigated (Goodwin et al., 2006; Morsdorf et al., 2008; Næsset, 2009). On the other hand, ULS and ALS systems differ significantly with regard to the measurement process (Morsdorf et al., 2018), i.e. regarding beam divergence, laser wavelength, pulse energy, pulse repetition frequency, scan angle, and flight altitude, depending on the deployed laser scanner, flight planing and platform. These parameters, however, were found to affect the point densities (Hopkinson, 2007; Morsdorf et al., 2008; Yu et al., 2004) and the point distributions across height (Hopkinson, 2007; Wieser et al., 2016) and, thus, have to be taken into account when FI-metrics derived from point clouds from different sensor systems are compared (Morsdorf et al., 2018).

In our study, we therefore address the comparability of four metrics that were computed from ULS and from ALS data and that describe the forest structure: height quantiles $h 10, h 50, h 90$, the fractional cover $f c$, the vegetation complexity index $V C I$, and the number of canopy layers nLayers. The metrics were chosen such that each metrics exploits the point cloud information in a different manner. They also differ in the concepts their computation bases on. The computation of the height quantiles is performed directly on the point cloud. $f c$ rests upon the the BeerLambert-law on canopy transmittance where the path length of the laser beam through the canopy layer is evaluated (Hopkinson and Chasmer, 2009; Weiss et al., 2004). The computation of $f c$, however, can be approximated through a classification of the point cloud into canopy points and non-canopy points and by subsequently forming their ratio (Morsdorf et al., 2006). For the calculation of the $V C I$, the point cloud is transformed into a voxel structure and the point cloud information is reduced by summarizing the point counts per voxel (Morsdorf et al., 2018; Pearse 
et al., 2018). The computation of nLayers, finally, marks a further generalization as the voxels are classified as "filled" if they are populated by points and as "empty" otherwise (Leiterer et al., 2015b).

The first aim of our study is to compare the scene coverage between ULS and ALS. The second aim is to investigate whether the differences in the point clouds and the different use of the point cloud information in the metrics computation affect the derived forest structure metrics. Our goals are to detect differences in the four metrics between ULS and ALS, analyse how the differences relate to the scale on which the metrics are computed and discuss which metrics are comparable between the two acquisition systems. The focus of our study therefore is to investigate how the differences in the point cloud information, and, along with this, the stated acquisition properties, which lead to these differences, can be reduced by selecting appropriate structure metrics in order to ensure the comparability between the two systems. Another aspect concerns how the differences in the deployed acquisition geometries and sensors, as well as corrections of the point cloud in the pre-processing of the point clouds, affect the calculated metrics. Such investigations, however, go beyond the scope of this study as no data sets with variances in the respective parameters were available.

\section{MATERIALS}

In this study, we selected two test sites near Düns, Vorarlberg, Austria. Test site 1 covers a $360 \times 360 \mathrm{~m}$ area centered at $5230390 \mathrm{~N}, 554860 \mathrm{E}(\mathrm{UTM} 32 \mathrm{~N})$, test site 2 covers a $240 \times 360 \mathrm{~m}$ area centered at $5230480 \mathrm{~N}, 554470 \mathrm{E}(\mathrm{UTM}$ $32 \mathrm{~N})$. The elevation ranges from 850 to 1070 m.a.s.l. The test sites cover predominantly forested areas that are dominated by coniferous trees. The mean canopy heights are $19.0 \mathrm{~m}$ for site 1 and $16.5 \mathrm{~m}$ for site 2 . In both test sites, a single layered forest ( $n$ Layers $=1$, read from the ALS data set and using a cell size of $8 \mathrm{~m}$ ) is dominating, with significantly less cells with two and more layers. Mean $f c$ is 0.64 for site 1 and 0.63 for site 2, both read from ALS data at cell sizes of $2 \mathrm{~m}$.

For the study area, we had an ALS and an ULS data set available, along with a forest map.

\subsection{ALS data}

The ALS data was acquired and provided by the federal state of Vorarlberg. The sites are covered by 6 (site 1) and 5 (site 2) flight strips, acquired between March and May 2011 under snow free conditions. The acquisition was performed using an Optech ALTM Gemini sensor. The used flight strips are derived from three different acquisition campaigns with respective average flight altitudes of 560, 700 and $760 \mathrm{~m}$ above ground. The sensor operates with a laser wavelength of $1064 \mathrm{~nm}$ and a beam divergence of $0.25 \mathrm{~nm}$ (Joerg et al., 2015), what results in footprint sizes between $14-19 \mathrm{~cm}$ for the given flight strips. The scan angles range in $\pm 30^{\circ}$. The average point densities within the evaluated forest areas are 33 and $23 \mathrm{pts} / \mathrm{m}^{2}$ for site 1 and 2 , respectively.

\subsection{ULS data}

ULS data was acquired in May 2014 using a Scout B1-100 ULS Helicopter system with a mounted Riegl VUX-1 sensor. Sites 1 and 2 were covered by 8 and 6 ULS-flight strips, respectively. The average flight altitude was $120 \mathrm{~m}$ above ground, ranging between 80 and $200 \mathrm{~m}$. The sensor operates with a wavelength of
$1550 \mathrm{~nm}$ and a beam divergence of $0.5 \mathrm{mrad}$ (Mandlburger et al., 2015). This results in footprint sizes of $4-10 \mathrm{~cm}$ on the ground for the chosen flight geometry. The scan angle was limited to $\pm 90^{\circ}$. The average point densities within the evaluated forest areas are 906 and $689 \mathrm{pts} / \mathrm{m}^{2}$ for site 1 and 2, respectively.

\subsection{Selection of the test site}

In order to facilitate a stable forest metrics computation, two measures were necessary. Firstly, we restricted the evaluation to forested areas according to the forest map. Due to the considerable time span between the ALS and the ULS acquisitions, however, major changes in the forest structure, i.e. logging activities, are discernible in some parts of the test sites. Secondly, in order to minimize a bias introduced by such changes in the forest structure, we limited the evaluation to raster cells with differences $\leq 1.0 \mathrm{~m}$ in the canopy height models (CHM) as computed from the ALS and ULS data.

The ALS data set is combined from two acquisition dates. While the second ALS acquisition was performed during the same phenological period as the ULS acquisition, i.e. under leaf-on conditions, the earlier ALS campaign was flown under leaf-off conditions. However, the vegetation predominantly comprises of conifer trees. The coniferous forest type, apart from the spatial selection, further warrants the comparability of the forest structure between the two data sets and acquisition dates.

\section{METHODS}

\subsection{Acquisition characteristics}

ALS and ULS data sets were investigated regarding differences in the point height distributions in the canopy layers and regarding the amount of occlusions occurring within the canopy. The latter investigation was performed through ray-tracing using the voxel traversal algorithm implemented in Kükenbrink et al. (2017).

\subsection{Structure metrics}

We computed height quantiles measuring the height of the $10 \%$ ( $h 10)$, the $50 \%$ - $(h 50)$ and the $90 \%$-quantile $(h 90)$ of the vertical distribution of the points. In order to reduce a bias through ground points, we excluded points deriving from $<0.5 \mathrm{~m}$ above ground from the analysis.

The $f c$ is a dimensionless parameter in range [0,1]. It describes the fraction of ground covered by vegetation within a certain ground area and, according to Morsdorf et al. (2006), can be computed from LiDAR data as:

$$
\text { fractional cover }=\frac{N_{\text {canopy }}}{N_{\text {total }}}
$$

$N_{\text {canopy }}$ thereby corresponds to the number of canopy points and $N_{\text {total }}$ to the total number of points within the area, where we selected a canopy threshold of $3 \mathrm{~m}$ above terrain for the discrimination of canopy from non-canopy points.

$V C I$ describes the evenness of the vertical distribution of the points across the canopy layers (Pearse et al., 2018; van Ewijk et al., 2011). Following these studies, it is calculated as:

$$
V C I=\frac{-\sum_{i=1}^{H} p_{i} \cdot \ln \left(p_{i}\right)}{\ln (H)}
$$


The canopy is divided into $H$ layers of a certain bin height, which we set to $1 \mathrm{~m}$ as was proposed by van Ewijk et al. (2011) and Pearse et al. (2018). $H$ is a constant value for each test site and was chosen such that the top point in the canopy height model lies in the uppermost layer. $p_{i}$ corresponds to the relative abundance of points in height layer $i$ with regard to the total number of points in the respective height column. $V C I$ is in range $[0,1]$ where $V C I=1$ signifies an even distribution of the points across the canopy height and decreasing values state an increasingly uneven distribution (van Ewijk et al., 2011).

We computed nLayers as described in Leiterer et al. (2015b). For the computation, the vertical point distributions are transformed to relative-frequency histograms, for which we again chose a bin height of $1 \mathrm{~m}$. The height bins then are classified as "filled" if their relative abundance is $\geq 1 \%$ or "empty" otherwise. As proposed by Leiterer et al. (2015b), isolated empty bins were reclassified to filled and vice versa. The number of layers corresponds to the number of changes from empty to filled bins in the classified height profiles.

\subsection{Evaluation of scale differences}

The described structure metrics were computed for raster cell sizes of $0.5,1,2,3,4,5,6,8$ and $10 \mathrm{~m}$, respectively. The layer width for the computation of $V C I$ and $n$ Layers, however, was left unchanged at $1 \mathrm{~m}$.

\section{RESULTS}

\subsection{Acquisition characteristics}

Figure 1 shows two $14 \times 10 \mathrm{~m}$ subsets from the ALS and the ULS data set, respectively, taken at the original resolutions of the respective point clouds. From visual inspection, one can recognize the high level of detail in the ULS point cloud, where tree stems and even the main branches are discernible. Figure 2 reports the point distributions across height, measured as point frequencies per height bin above the terrain, for the ALS and the ULS point cloud. The ULS acquisition shows a higher fraction of points from the canopy than ALS, particularly within medium and lower canopy layers. In the sub-canopy layer (i.e. below the canopy threshold of $3 \mathrm{~m}$ as used in our study), on the other hand, the relative fractions of total points in the ALS data are equal to or larger than in the ULS data. Furthermore, ALS shows a better relative coverage of the ground than ULS with $33.8-36.2 \%$ of the ALS points deriving from below $1 \mathrm{~m}$ above ground while the respective fractions of ULS points are 14.7-21.3\%.

Table 1 reports the fraction of observed voxels (fObserved), i.e. voxels within the canopy that have been traversed by the laser and that either contain points or not, and the fraction of occluded voxels (fOccluded), i.e. voxels that theoretically lie in the laser beam path but that are not reached by the beam due to occlusions through canopy material. For voxels with side lengths of $0.5 \mathrm{~m}$, the fraction of occluded voxels amounts to $15.8-31.4 \%$ for ALS and to $2.7-5.4 \%$ for ULS. The occlusions decrease with increasing voxel sizes.

Figure 3 depicts the fraction of occluded voxels in dependence of the voxel location above ground for a voxel size of $1 \mathrm{~m}$. The fractions are in regard to the total number of occluded voxels in the respective data sets. The occlusion profiles reveal occlusions in the ULS system to occur predominantly within lower canopy layers while for the ALS system, already medium canopy layers are prone to the occlusion effect.

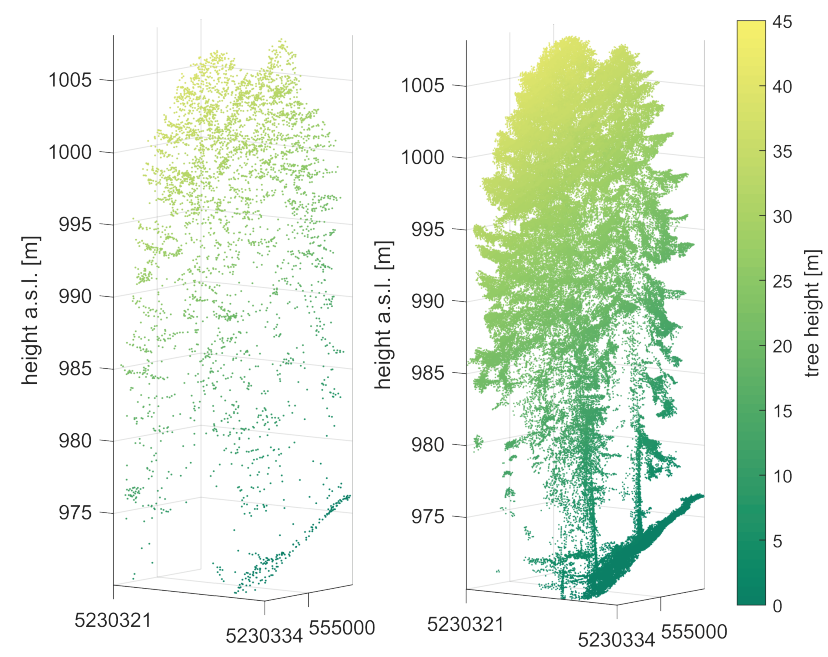

Figure 1. Oblique view onto a 14 x $10 \mathrm{~m}$ (northing x easting) subset of site 1, taken from the ALS (left) and the ULS (right) point cloud. The subsets are centered at $5230328.5 \mathrm{~N}, 555000 \mathrm{E}$ and were taken at the original resolutions of the respective point clouds.
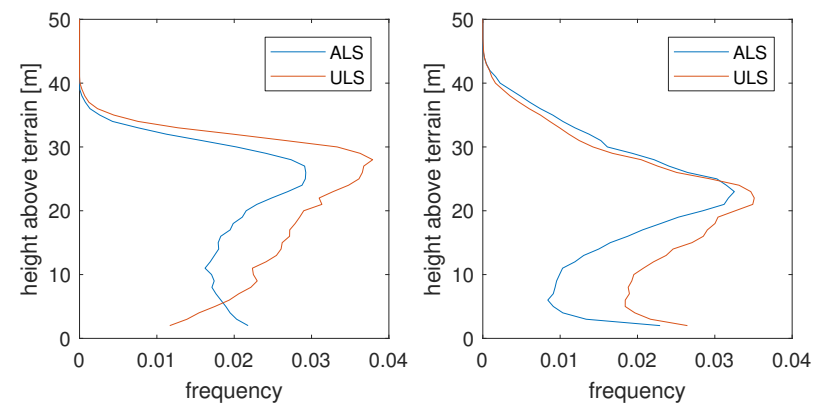

Figure 2. Comparison of point frequencies depending on height from ALS and ULS data for site 1 (left) and site 2 (right). The frequencies are measured in $1 \mathrm{~m}$ height intervals and state the fraction of points with regard to the total number of points per point cloud. As the bottom most layer contains the largest

fraction of points, it has been excluded for a clearer representation.

\subsection{Structure metrics differences}

Figure 4 shows the mean differences between the structure metrics derived from the ALS and the ULS data sets in dependence of the cell sizes on basis of which the metrics have been calculated. The means were calculated from the absolute differences, i.e. without taking into account the direction of the differences. Height quantile differences are measured as absolute values in $[\mathrm{m}]$ (mean difference) and as relative difference with regard to the maximum canopy height within the cell (mean relative difference). $f c$ and $V C I$ are measured in the value range of these two metrics, which corresponds to [0,1]. The mean difference for nLayers is stated in number of layers, where the maximum number of layers was 5 in site 1 and 6 in site 2, respectively. Histograms of the absolute differences for $h 90, f c$ and nLayers are depicted in Figures 5, 6 and 7, respectively.

Of the height quantile metrics, $h 10$ shows the largest differences between ALS and ULS, however, the differences get to the range of differences in $h 50$ in terms of absolute mean distances $(5.9-2.9 \mathrm{~m}$ for $h 10 ; 3.6-3.1 \mathrm{~m}$ for $h 50)$ and decrease be- 

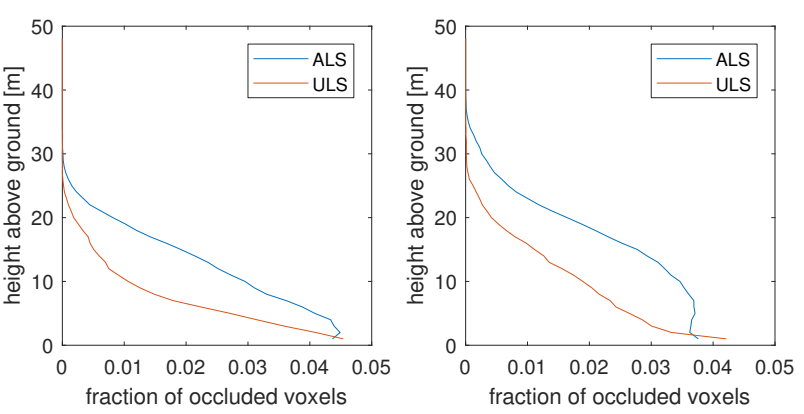

Figure 3. Fraction of occluded voxels in dependence of height above ground for the ALS and the ULS acquisitions. The plots show the occlusions for raster cell sizes of $1 \mathrm{~m}$ for site 1 (left) and site 2 (right). The reported fractions are relative to the total number of occluded voxels within the respective data set, which are reported in Table 1

Table 1. Observation statistics for site 1 (top) and site 2 (bottom), stating the fraction of observed voxels ([\%], fObserved) and the fraction of occluded voxels ([\%], fOccluded). The voxels have a squared ground area with side length stated by Cell size and a constant voxel height of $1 \mathrm{~m}$.

\begin{tabular}{lcccc}
\hline \multirow{2}{*}{$\begin{array}{l}\text { Cell } \\
\text { size }\end{array}$} & \multicolumn{2}{c}{ fObserved } & \multicolumn{2}{c}{ fOccluded } \\
\cline { 2 - 5 } & ALS & ULS & ALS & ULS \\
\hline $0.5 \mathrm{~m}$ & 84.2 & 97.3 & 15.8 & 2.7 \\
$1 \mathrm{~m}$ & 94.4 & 98.2 & 5.6 & 1.8 \\
$2 \mathrm{~m}$ & 97.6 & 99.8 & 2.4 & 0.2 \\
$3 \mathrm{~m}$ & 99.4 & $>99.9$ & 0.6 & $<0.01$ \\
$4 \mathrm{~m}$ & 99.9 & 100.0 & 0.1 & 0.0 \\
$5 \mathrm{~m}$ & 100.0 & 100.0 & 0.0 & 0.0 \\
\hline $0.5 \mathrm{~m}$ & 68.6 & 94.6 & 31.4 & 5.4 \\
$1 \mathrm{~m}$ & 86.8 & 96.6 & 13.2 & 3.4 \\
$2 \mathrm{~m}$ & 95.1 & 99.4 & 4.9 & 0.6 \\
$3 \mathrm{~m}$ & 98.7 & 99.9 & 1.3 & 0.1 \\
$4 \mathrm{~m}$ & 99.7 & $>99.9$ & 0.3 & $<0.01$ \\
$5 \mathrm{~m}$ & 99.9 & $>99.9$ & 0.1 & $<0.01$ \\
$6 \mathrm{~m}$ & $>99.9$ & $>99.9$ & $<0.01$ & $<0.01$ \\
$8 \mathrm{~m}$ & 100.0 & 100.0 & 0.0 & 0.0 \\
\hline
\end{tabular}

low the values of the latter in terms of the relative mean difference $(0.29-0.11$ for $h 10 ; 0.19-0.14$ for $h 50)$ for increasing cell sizes. Differences between the systems in $h 90$ are smaller in general (mean differences of 3.4-2.2 m; mean relative differences of $0.15-0.07)$. On average, the ALS heights are lower than the ULS heights for all three quantiles (exemplary shown in Figure 5 for the case of differences in $h 90)$. With increasing cell sizes, $h 10$ shows a clear decrease in both, the mean difference and the mean relative difference. For $h 50$ and $h 90$, the respective changes in the mean differences are small with increasing cell sizes. On the other hand, if the cell sizes are increased, the mean relative differences between the systems slightly decrease for $h 50$, but increase for $h 90$. However, for $h 50$ and $h 90$, changes of the mean relative differences are less pronounced than the respective changes in $h 10$.

$f c$ shows large differences between ALS and ULS for all cell sizes with mean differences between $0.19-0.24$, depending on the cell size. $f c$ on average is higher from ULS than from ALS, leading to a right-skewed distribution of differences in the histogram in Figure 6. Furthermore, the mean differences between ALS and
ULS increase with increasing cell sizes.

$V C I$, on the other hand, shows differences between the systems in the range of $0.03-0.11$ with a distinct decrease of the differences with increasing cell sizes.

The mean difference in nLayers is below one layer, signifying that on average, metrics from both data sets are comparable. The difference histograms of nLayers (Figure 7) further illustrate the relative number of pixels with no differences in nLayers to increase from $54.5 \%$ and $55.5 \%$ to $76.9 \%$ and $88.7 \%$ for sites 1 and 2 , respectively, when increasing the raster sizes from $1 \mathrm{~m}$ to $10 \mathrm{~m}$.

\section{DISCUSSION}

\subsection{Acquisition characteristics}

Studies by Hopkinson (2007), Gaveau and Hill (2003) and Wieser et al. (2016) discussed the laser beam penetration into the canopy to depend on sensor characteristics (e.g. laser pulse power, beam divergence, wavelength), on acquisition parameters (flight altitude and pulse repetition frequency), and on canopy characteristics. We found the largest discrepancies in the point height distributions between ALS and ULS to occur within medium and lower canopy layers (Figure 2), what we ascribe to the larger beam width of ALS and variations in the laser pulse power between the system, considering the findings by Hopkinson (2007). However, due to a missing broader data basis, we could not further investigate the respective impacts of the acquisition characteristics and sensor properties.

Table 1 reports the completeness of scene coverage in terms of the fraction of observed voxels to be higher by the factor of 5 to 6 for the ULS compared to the ALS system for $0.5 \mathrm{~m}$ voxel cells (15.8-31.4\% occluded voxels for ALS, 2.7-5.4\% occluded voxels for ULS) while the completeness of scene coverage becomes similar for voxels with side length $\geq 4 \mathrm{~m}$. Kükenbrink et al. (2017) found the fraction of occluded voxels to become very low for voxel sizes exceeding this value. However, we used voxels with a constant voxel height of $1 \mathrm{~m}$ while Kükenbrink et al. (2017) used cubic voxels.

Morsdorf et al. (2018) and Schneider et al. (2019) showed occlusion profiles of ULS measurements and discussed the number of occluded voxels to increase on decreasing canopy height levels. Furthermore, the amount of occluded voxels was found to be higher for the same acquisition characteristics under leaf-on than under leaf-off conditions (Kükenbrink et al., 2015; Morsdorf et al., 2018). On the other hand, Kükenbrink et al. (2017) discussed the magnitude of occlusion effects to decrease with higher pulse densities and if the scene is covered under multiple scan angles. These latter acquisition characteristics differed significantly between the two sensor systems deployed in this study, what could explain our results.

\subsection{Structure metrics differences}

Differences in the height quantiles between ULS and ALS point clouds diverge with increasing penetration into the canopy. As depicted exemplary for $h 90$ in Figure 5, ALS generally underestimates the respective height quantiles compared to ULS. Similar findings for differences in the height estimates from the two systems were reported in Jaakkola et al. (2010), Wallace et al. (2014) and Wieser et al. (2016). Underestimations of height metrics from ALS have been discussed in previous studies, which attributed them to sensor and acquisition characteristics, with the result that ALS is likely to miss tree tops (Gaveau and Hill, 2003; 

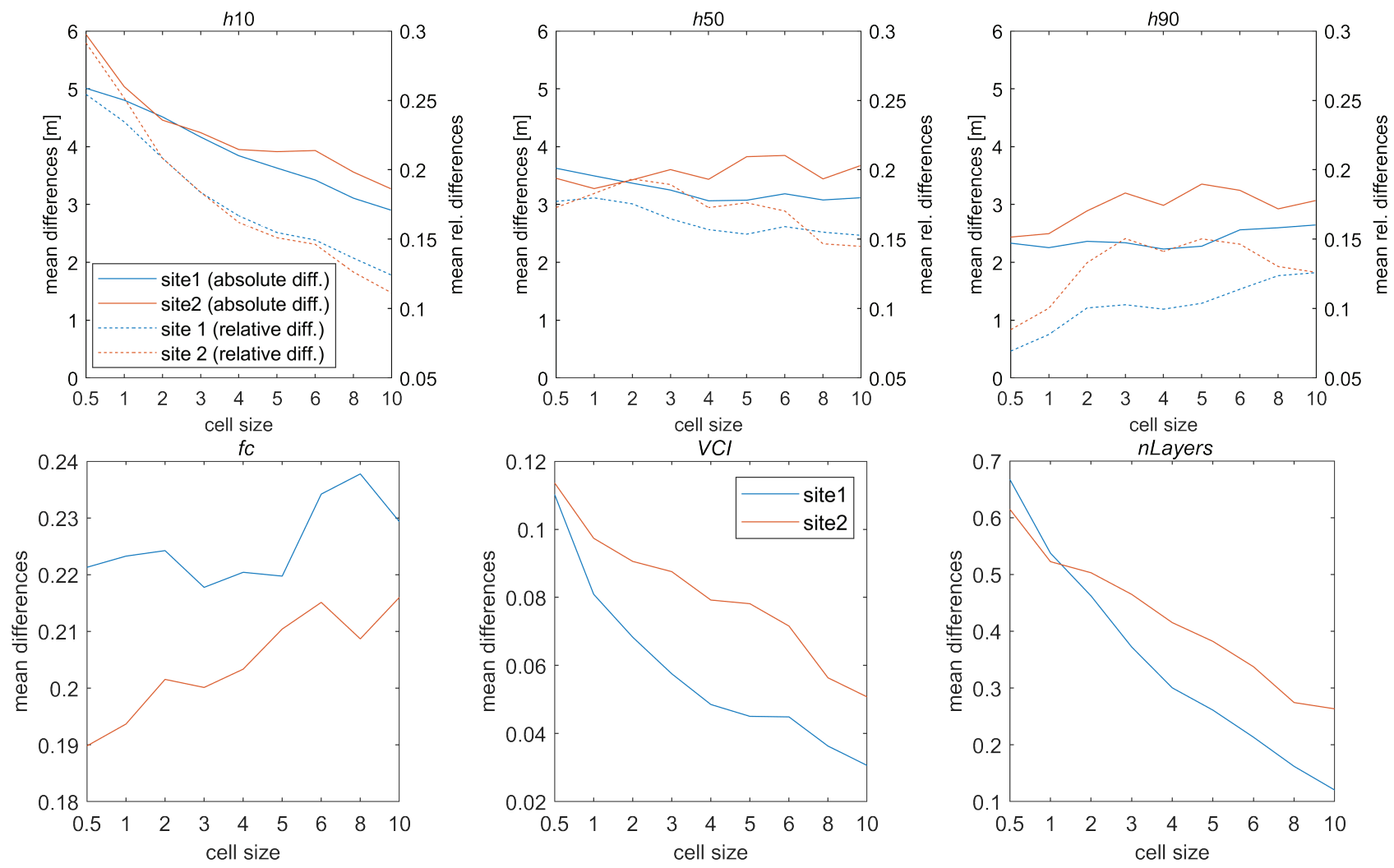

Figure 4. Top row, left to right: Mean differences (solid lines, left y-label) and mean relative differences (dashed lines, right y-label) in $h 10, h 50, h 90$ from ULS and ALS as function of the cell size for which the metrics were computed. Bottom row, left to right: Mean differences in $f c, V C I$, nLayers from ULS and ALS.
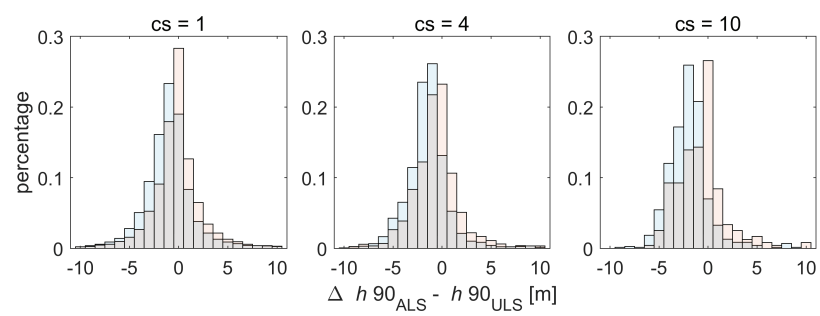

Figure 5. Relative histogram of differences between $h 90$ calculated from ULS data with regard to ALS data for raster cell sizes $(c s)$ of 1,4 , and $10 \mathrm{~m}$, respectively. Bin widths are set to

$1 \mathrm{~m}$, differences are measured in [m]. Orange: site 1, blue: site 2, gray: overlay of the histograms.
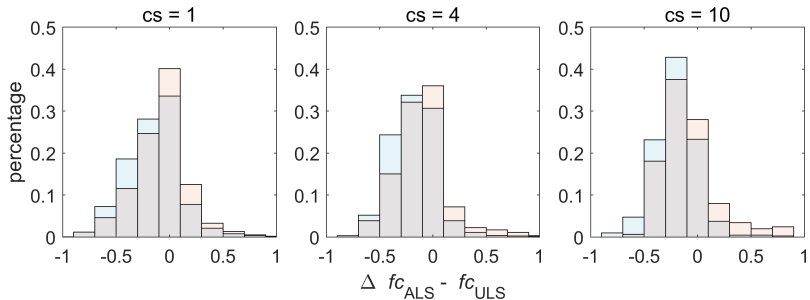

Figure 6. Relative histogram of differences between $f c$ calculated from ULS data with regard to ALS data for raster cell sizes $(c s)$ of 1,4 , and $10 \mathrm{~m}$, respectively. Bin widths are set to $\Delta f c=0.2$, differences are measured in the unit of $f c$. Orange: site 1, blue: site 2 , gray: overlay of the histograms.

puted from ALS data to reference data for a circular area with a $2 \mathrm{~m}$ radius. Our results revealed the smallest differences between ULS and ALS to coincide with smaller cell sizes while the differences increased with larger cells. Overall, however, the large differences between the systems make the comparability of the metrics questionable for all cell sizes.

In $V C I$, we found small differences between the systems and differences became smaller when computed for larger cell sizes. The evaluation of $V C I$ on larger scales, thus, ensures comparability of the metrics and could likely be increased beyond the level reported by us if it is computed for areas of $400 \mathrm{~m}^{2}$ as in van Ewijk et al. (2011).

nLayers computed from ULS and ALS shows a high level of comparability for large cell sizes. Leiterer et al. (2015a) reported a saturation in the information content with regard to the point 

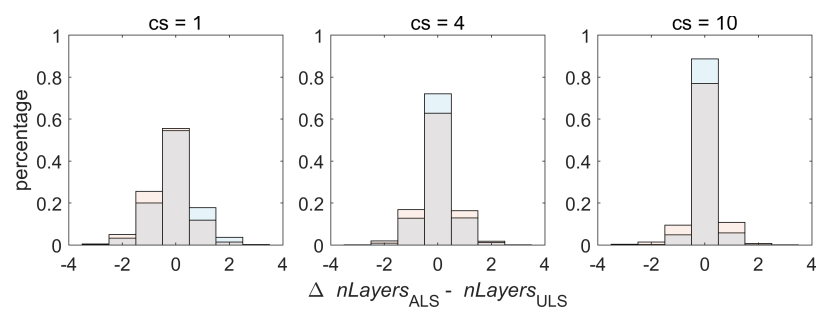

Figure 7. Relative histograms of differences between $n$ Layers calculated from ULS data with regard to ALS data for raster cell sizes $(c s)$ of 1,4 , and $10 \mathrm{~m}$, respectively. Bin widths are set to 1 , corresponding to one layer. Orange: site 1, blue: site 2, gray: overlay of the histograms.

density for the way the point cloud information is used for the computation of nLayers. Leiterer et al. (2015a) and Leiterer et al. $(2015 b)$ therefore stated raster sizes of $8-10 \mathrm{~m}$ to be the most appropriate choice for canopy layer characterizations. As further shown in Table 1, we can assume a similar completeness of scene coverage in terms of fraction of observed voxels for such cell sizes.

Although we could define a scale on which the metrics from ULS and ALS become comparable, a more fundamental consideration regards the reasonability of comparing metrics derived form the two systems. ALS point clouds are relatively sparse (Figure 1, left), requiring empirical approaches to relate predictor variables computed from the point cloud, as e.g. height quantiles, to field inventoried parameters (Næsset, 1997; Næsset, 2002). ULS, on the other hand, provides very high point densities and, among with this, a high level of detail, which potentially allows for the recognition of the tree architecture up to the level of single branches (Figure 1, right). This difference in the information content of ULS data has been discussed in Morsdorf et al. (2018) and the authors put into question the usability of empirical approaches known from ALS for the application on ULS data. Although their scepticism, we believe that metrics from ULS data can be used in a similar way as metrics from ALS data, as long as one can prove the derived metrics to be comparable from statistical analyses. However, with simple empirical approaches, we neglect the high information content that ULS provides. The integration of ULS data into forest inventories therefore requires further investigations in order to fully exploit the high information content that ULS provides.

On the other hand, the level of detail, which is required by the stakeholders in forestry, is on the scale of several meters (Leiterer et al., 2015b). The future task, thus, will be to bring the high level of detail from ULS to broader extents.

\subsection{Implications on the metrics selection}

Our results highlight a number of findings that are relevant for the selection of metrics with high comparabilities between ULS and ALS. i) we found that the capability of the systems to penetrate into the canopy layer impacts the comparability of metrics directly computed from the point cloud. In our study, the canopy penetration varies between ULS and ALS, what leads to differences in the height quantile metrics. ii) we found that the theoretical concepts, which underlie the computation of structure metrics, have to be thoroughly considered in order to evaluate properties which are measured in the same way. Exemplary for such a metric, we evaluated $f c$ based on the approximation by Morsdorf et al. (2006). From a conceptual perspective, however, $f c$ rests upon the evaluation of the canopy transmittance (Weiss et al., 2004). As Korhonen and Morsdorf (2014) discussed, the implemented approximation in the computation step therefore only applies to nearly nadir-looking acquisition geometries. For ULS, this assumption is violated due to the large off-nadir scan angles and the measured transmittance from ULS is not comparable to the one from ALS. iii) we found that voxel-based metrics ( $V C I$ in our case) increased the comparability of metrics from different sensor systems. Our finding is consistent to the results by Pearse et al. (2018) who illustrated the benefit of voxel-based forest metrics from ALS data. Furthermore, we found that a higher level of generalization of the point cloud information within the voxels further reduced metrics differences between the systems (nLayers).

However, for a more robust generalization of our findings, we propose to investigate more systematically the impact of the acquisition and sensor characteristics, which are specific to ULS and ALS campaigns, respectively, on the resulting point clouds and the derived structure metrics. This requires to capture the same scene with different flight schemes, LiDAR sensor systems, and scanning properties. As we currently only had the used data sets available, we could not further assess the impacts of these parameters. We assume that our findings regarding the metrics differences are valid for ULS acquisitions similar to ours. The term ULS, however, does not define the acquisition geometry in a strict way. In our case, the flying altitude above ground ranged from 80 to $200 \mathrm{~m}$, while other studies reported altitudes of $15 \mathrm{~m}$ (Wieser et al., 2017) to $30 \mathrm{~m}$ (Morsdorf et al., 2017) above the canopy top height. We assume that acquisition geometries change drastically, depending on whether the UAV is flown almost on crown top height or tens of meters above.

\section{SUMMARY AND CONCLUSIONS}

We investigated differences in the point clouds from ULS and ALS and analyzed the comparability of forest structure metrics computed from the respective data sets as function of scale, for which we tested cell sizes with side length of $0.5-10 \mathrm{~m}$ for the metrics computation. We found differences of the completeness of scene coverage to be levelled out between ULS and ALS for horizontal voxel side lengths $\geq 4 \mathrm{~m}$. The point distribution within the canopy differed between the two systems with ULS having a higher relative coverage of medium to lower canopy layers compared to ALS, and ALS having a higher fraction of ground points. From the compared structure metrics, we found $V C I$ and nLayers to provide comparable estimates from ULS and ALS and propose cell sizes of 8-10 $\mathrm{m}$ for their computation. On the other hand, $h 10$ and $h 50$ showed larger mean relative differences, what, in our view, makes the direct comparison of these metrics questionable. The differences in $h 90$ were smaller, but the metrics was generally underestimated from ALS data. $f c$, finally, showed huge differences between ULS and ALS why we negate the comparability of this metrics between ULS and ALS.

From our reported findings, we further conclude that the means by which the point cloud information is utilized in the structure metrics computation has an influence on the comparability of ULS and ALS metrics. A higher level of generalization of the point cloud information through voxelization resulted in smaller metrics differences between ULS and ALS (VCI) than when the untransformed point cloud was used $(h 10, h 50, h 90)$. The level of comparability was further increased by the generalization of the point counts per voxel cell into the information on whether a voxel cell was populated or not (nLayers). In contrast, $f c$ revealed large differences between ULS and ALS, although its computa- 
tion bases on a coarse partition of the point cloud into canopy and non-canopy points only. We hypothetize the cause to be in the large scan angle differences between ULS and ALS systems. Finally, our results suggest, that the comparability of the metrics increases with larger cell sizes. From other studies, however, we learn that this statement does not apply to all metrics. Moreover, we suspect that beyond a certain cell size, the metrics loose their meaning. Therefore, further research is required on how ALS and ULS data can be integrated jointly into forest inventories, particularly making better use of the high level of detail offered by ULS.

\section{References}

Gaveau, D. L. and Hill, R. A., 2003. Quantifying canopy height underestimation by laser pulse penetration in small-footprint airborne laser scanning data. Canadian Journal of Remote Sensing 29(5), pp. 650-657.

Goodbody, T. R., Coops, N. C., Marshall, P. L., Tompalski, P. and Crawford, P., 2017. Unmanned aerial systems for precision forest inventory purposes: A review and case study. The Forestry Chronicle 93(1), pp. 71-81.

Goodwin, N. R., Coops, N. C. and Culvenor, D. S., 2006. Assessment of forest structure with airborne LiDAR and the effects of platform altitude. Remote Sensing of Environment 103(2), pp. $140-152$.

Guo, Q., Su, Y., Hu, T., Zhao, X., Wu, F., Li, Y., Liu, J., Chen, L., Xu, G., Lin, G. et al., 2017. An integrated UAV-borne lidar system for 3D habitat mapping in three forest ecosystems across China. International Journal of Remote Sensing 38(810), pp. 2954-2972.

Hollaus, M., 2015. 3d point clouds for forestry applications. Österreichische Zeitschrift für Vermessung und Geoinformation (VGI) 103(2+3), pp. 138-150.

Hopkinson, C., 2007. The influence of flying altitude, beam divergence, and pulse repetition frequency on laser pulse return intensity and canopy frequency distribution. Canadian Journal of Remote Sensing 33(4), pp. 312-324.

Hopkinson, C. and Chasmer, L., 2009. Testing LiDAR models of fractional cover across multiple forest ecozones. Remote Sensing of Environment 113(1), pp. 275 - 288.

Jaakkola, A., Hyyppä, J., Kukko, A., Yu, X., Kaartinen, H., Lehtomäki, M. and Lin, Y., 2010. A low-cost multi-sensoral mobile mapping system and its feasibility for tree measurements. ISPRS journal of Photogrammetry and Remote Sensing 65(6), pp. 514-522.

Joerg, P. C., Weyermann, J., Morsdorf, F., Zemp, M. and Schaepman, M. E., 2015. Computation of a distributed glacier surface albedo proxy using airborne laser scanning intensity data and in-situ spectro-radiometric measurements. Remote Sensing of Environment 160, pp. 31-42.

Korhonen, L. and Morsdorf, F., 2014. Estimation of canopy cover, gap fraction and leaf area index with airborne laser scanning. In: M. Maltamo, E. Næsset and J. Vauhkonen (eds), Forestry Applications of Airborne Laser Scanning: Concepts and Case Studies. Managing Forest Ecosystems, 27, Springer, The Netherlands. 464pp.

Kükenbrink, D., Leiterer, R., Schneider, F. D., Schaepman, M. E. and Morsdorf, F., 2015. Voxel based occlusion mapping and plant area index estimation from airborne laser scanning data. Proceedings of SilviLaser 28-30 September 2015, La Grande Motte, France, pp. 232 - 234.
Kükenbrink, D., Schneider, F. D., Leiterer, R., Schaepman, M. E. and Morsdorf, F., 2017. Quantification of hidden canopy volume of airborne laser scanning data using a voxel traversal algorithm. Remote Sensing of Environment 194, pp. 424-436.

Leiterer, R., Furrer, R., Schaepman, M. E. and Morsdorf, F., 2015a. Forest canopy-structure characterization: A data-driven approach. Forest Ecology and Management 358, pp. 48-61.

Leiterer, R., Torabzadeh, H., Furrer, R., Schaepman, M. E. and Morsdorf, F., 2015b. Towards Automated Characterization of Canopy Layering in Mixed Temperate Forests Using Airborne Laser Scanning. Forests 6(11), pp. 4146-4167.

Lin, Y., Hyyppa, J. and Jaakkola, A., 2011. Mini-UAV-borne LIDAR for fine-scale mapping. IEEE Geoscience and Remote Sensing Letters 8(3), pp. 426-430.

Mandlburger, G., Pfennigbauer, M., Riegl, U., Haring, A., Wieser, M., Glira, P. and Winiwarter, L., 2015. Complementing airborne laser bathymetry with uav-based lidar for capturing alluvial landscapes. Proceedings of SPIE 9637, pp. 96370A.

Morsdorf, F., Eck, C., Zgraggen, C., Imbach, B., Schneider, F. D. and Kükenbrink, D., 2017. UAV-based LiDAR acquisition for the derivation of high-resolution forest and ground information. The Leading Edge 36(7), pp. 566-570.

Morsdorf, F., Frey, O., Meier, E., Itten, K. I. and Allgöwer, B., 2008. Assessment of the influence of flying altitude and scan angle on biophysical vegetation products derived from airborne laser scanning. International Journal of Remote Sensing 29(5), pp. 1387-1406.

Morsdorf, F., Kükenbrink, D., Schneider, F., Abegg, M. and Schaepman, M., 2018. Close-range laser scanning in forests: towards physically based semantics across scales. Interface Focus 8(2), pp. 20170046.

Morsdorf, F., Ktz, B., Meier, E., Itten, K. and Allgwer, B., 2006. Estimation of LAI and fractional cover from small footprint airborne laser scanning data based on gap fraction. Remote Sensing of Environment 104(1), pp. 50 - 61.

Næsset, E., 1997. Estimating timber volume of forest stands using airborne laser scanner data. Remote Sensing of Environment 61(2), pp. $246-253$.

Næsset, E., 2002. Predicting forest stand characteristics with airborne scanning laser using a practical two-stage procedure and field data. Remote Sensing of Environment 80(1), pp. 88-99.

Næsset, E., 2009. Effects of different sensors, flying altitudes, and pulse repetition frequencies on forest canopy metrics and biophysical stand properties derived from small-footprint airborne laser data. Remote Sensing of Environment 113(1), pp. $148-159$.

Næsset, E., 2014. Area-based inventory in norway from innovation to an operational reality. In: M. Maltamo, E. Næsset and J. Vauhkonen (eds), Forestry Applications of Airborne Laser Scanning: Concepts and Case Studies. Managing Forest Ecosystems, 27, Springer, The Netherlands. 464pp, pp. 215240.

Pearse, G. D., Watt, M. S., Dash, J. P., Stone, C. and Caccamo, G., 2018. Comparison of models describing forest inventory attributes using standard and voxel-based lidar predictors across a range of pulse densities. International Journal of Applied Earth Observation and Geoinformation. 
Schneider, F. D., Kükenbrink, D., Schaepman, M. E., Schimel, D. S. and Morsdorf, F., 2019. Quantifying 3D structure and occlusion in dense tropical and temperate forests using close-range LiDAR. Agricultural and Forest Meteorology 268, pp. 249-257.

van Ewijk, K. Y., Treitz, P. M. and Scott, N. A., 2011. Characterizing forest succession in Central Ontario using LiDARderived indices. Photogrammetric Engineering \& Remote Sensing 77(3), pp. 261-269.

Vauhkonen, J., Maltamo, M., McRoberts, R. E. and Næsset, E., 2014. Introduction to Forestry Applications of Airborne Laser Scanning. In: M. Maltamo, E. Næsset and J. Vauhkonen (eds), Forestry Applications of Airborne Laser Scanning: Concepts and Case Studies. Managing Forest Ecosystems, 27, Springer, The Netherlands. 464pp, pp. 1-18.

Wallace, L., Lucieer, A. and Watson, C. S., 2014. Evaluating tree detection and segmentation routines on very high resolution UAV LiDAR data. IEEE Transactions on Geoscience and Remote Sensing 52(12), pp. 7619-7628.

Wallace, L., Lucieer, A., Malenovskỳ, Z., Turner, D. and Vopěnka, P., 2016. Assessment of forest structure using two UAV techniques: A comparison of airborne laser scanning and structure from motion (SfM) point clouds. Forests 7(3), pp. 62.

Weiss, M., Baret, F., Smith, G., Jonckheere, I. and Coppin, P., 2004. Review of methods for in situ leaf area index (LAI) determination: Part II. Estimation of LAI, errors and sampling. Agricultural and Forest Meteorology 121(12), pp. 37 - 53.

White, J. C., Coops, N. C., Wulder, M. A., Vastaranta, M., Hilker, T. and Tompalski, P., 2016. Remote sensing technologies for enhancing forest inventories: A review. Canadian Journal of Remote Sensing 42(5), pp. 619-641.

Wieser, M., Hollaus, M., Mandlburger, G., Glira, P. and Pfeifer, N., 2016. ULS LiDAR supported analyses of laser beam penetration from different ALS systems into vegetation. ISPRS Annals of Photogrammetry, Remote Sensing \& Spatial Information Sciences III-3, pp. 233-239.

Wieser, M., Mandlburger, G., Hollaus, M., Otepka, J., Glira, P. and Pfeifer, N., 2017. A case study of UAS borne laser scanning for measurement of tree stem diameter. Remote Sensing 9(11), pp. 1154.

Yu, X., Hyyppä, J., Hyyppä, H. and Maltamo, M., 2004. Effects of flight altitude on tree height estimation using airborne laser scanning. Proceedings of the Laser Scanners for Forest and Landscape Assessment-Instruments, Processing Methods and Applications, 3-6 October 2004, Freiburg, Germany. International Archives of Photogrammetry, Remote Sensing and Spatial Information Sciences XXXVI, 8/W2, pp. 96-101. 